INPLASY

PROTOCOL

To cite: Yan et al. Therapeutic effect and safety of Tuina on Sciatica: A protocol for systematic review and metaanalysis. Inplasy protocol 2021100034. doi:

10.37766/inplasy2021.10.0034

Received: 10 October 2021

Published: 10 October 2021

Corresponding author:

Yan Huixin

doctoryhx@126.com

Author Affiliation:

Yueyang Hospital of Integrated

Traditional Chinese and Western Medicine,Shanghai

University of Traditional

Chinese Medicine.

Support: SMC2013.

Review Stage at time of this submission: Preliminary searches.

Conflicts of interest:

None declared.

\section{Therapeutic effect and safety of Tuina on Sciatica: A protocol for systematic review and meta-analysis}

\author{
Yan, HX'; An, Y2; Zhang, T3; Zhao, JN4; Yan, JT5.
}

Review question / Objective: This study aimed to conduct a meta-analysis of Tuina in the treatment of Sciatica to clarify its efficacy and safety.

Condition being studied: Sciatica is one of the common pain symptoms in the human body, also known as radiating leg pain. Sciatica is increasingly occurring due to poor posture and lack of physical exercise all over the world. At present, many studies have indicated that Tuina can improve the clinical symptoms and functional status of sciatica. However, there is currently no relevant systematic review to evaluate and report this clinical scientific issue. Consequently, this study will conduct a meta-analysis on the effectiveness and safety of Tuina therapy for sciatica.

INPLASY registration number: This protocol was registered with the International Platform of Registered Systematic Review and Meta-Analysis Protocols (INPLASY) on 10 October 2021 and was last updated on 10 October 2021 (registration number INPLASY2021100034).

\section{INTRODUCTION}

Review question / Objective: This study aimed to conduct a meta-analysis of Tuina in the treatment of Sciatica to clarify its efficacy and safety.

Condition being studied: Sciatica is one of the common pain symptoms in the human body, also known as radiating leg pain. Sciatica is increasingly occurring due to poor posture and lack of physical exercise all over the world. At present, many studies have indicated that Tuina can improve the clinical symptoms and functional status of sciatica. However, there is currently no relevant systematic review to evaluate and report this clinical scientific issue. 
Consequently, this study will conduct a meta-analysis on the effectiveness and safety of Tuina therapy for sciatica.

\section{METHODS}

Search strategy: Randomized controlled trials (RCTs) related to Tuina treatment of sciatica will be retrieved from the Chinese and English databases and Clinical Trial Register (CTR). These databases include China National Knowledge Infrastructure (CNKI), Wan Fang Database (Wang Fang), Chinese Biomedical Literature Database (CBM), VIP Database for Chinese Technical Periodicals (VIP), PubMed, Embase, Web of Science, Cochrane Library, and Medline, etc.We will consider articles published in English or Chinese between database initiation and October 2021.

Participant or population: Patients suffering from Sciatica.

Intervention: Simple Tuina therapy or Tuina therapy combined with other ordinary and basic treatment will be the intervention of the experimental group.

Comparator: If the control group is treated with Chinese herbal medication, placebo, western medication, acupuncture, acupoint injection, traction and so on, or even with no treatment, will be included.

Study designs to be included: This review will include randomized controlled trials (RCTs) on Tuina for sciatica published in Chinese and English language.

Eligibility criteria: Our study will include patients of any age group who have been diagnosed with sciatica.

Information sources: Randomized controlled trials (RCTs) related to Tuina treatment of sciatica will be retrieved from the Chinese and English databases and Clinical Trial Register (CTR). These databases include China National Knowledge Infrastructure (CNKI), Wan Fang Database (Wang Fang), Chinese Biomedical Literature Database (CBM), VIP Database for Chinese Technical Periodicals
(VIP), PubMed, Embase, Web of Science, Cochrane Library, and Medline, etc.We will consider articles published in English or Chinese between database initiation and October 2021.

Main outcome(s): The main outcomes of this review are clinical effective rate and visual analogue scale (VAS).

Additional outcome(s): The additional outcomes include pain threshold, Oswestry Disability Index (ODI), modified Japanese Orthopaedic Association (JOA) score, sixpoint behavior (BRS-6) score, six-point behavior (BRS-6) score, adverse reactions, etc.

Quality assessment / Risk of bias analysis: The researchers of our team will use the Cochrane Collaborative tool to independently assess the risk of bias in all final inclusion of trials. In each study, seven items (Random sequence generation, allocation concealment, blinding of participants and personnel, blindness of outcome assessments, incomplete outcome data, selective reporting, and other bias) will be evaluated as unclear, high, or low risk. The results of the bias assessment risk will be carefully checked by other researchers, and any disagreement will be finalized after further discussion by all investigators. If the number of included studies exceeds 10 trials, we will use Revman 5.3 software to generate funnel plots to report publication bias. When discovering asymmetrical funnel plot, we will try to explore and analyze the possible causes with the Egger method.

Strategy of data synthesis: Review Manager software (Revman, Version 5.3 for Windows) will be used to perform the metaanalysis. The Chi-Squared test and 12 statistic will be used to assess the heterogeneity of literature according to the values of $P$ and $\mathbf{I 2}$. If the homogeneity is low ( $P>0.1 ; 12<50 \%)$, the fixed-effect model will be used for the meta-analysis; otherwise, the random effect model will be selected. However, when the heterogeneity is particularly high, we will first try to find 
the cause of its occurrence, and then decide the final meta-analysis model to choose.

Subgroup analysis: Our team firstly will considered the subgroup analysis of primary sciatica and secondary sciatica to explore the influence of massage on different types of sciatica. If conditions permit, we will also consider subgroup analysis from the following factors such as the types of Tuina techniques, treatment acupoints and meridians, treatment time, intervention frequency, age, gender, region, sample size, etc.

Sensitivity analysis: Sensitivity analysis is also one of the indispensable methods to deal with heterogeneity. The sensitivity analysis, we will conduct, is to explore the impact of trial bias risk on preliminary results. If there is significant statistical heterogeneity, sensitivity analysis will exclude low-quality studies, and repeat meta-analysis based on sample size and insufficient data to assess quality and robustness.

Country(ies) involved: China.

Keywords: Tuina, sciatica, systematic review, meta-analysis, protocol.

Contributions of each author:

Author 1 - Yan Huixin.

Email: doctoryhx@126.com

Author 2 - An Yun.

Email: anyun920122@163.com

Author 3 - Zhao Tao.

Email: 510444994@qq.com

Author 4 - Zhao Jiangna.

Email: kfxszjn@163.com

Author 5 - Yan Juntao.

Email: doctoryjt@163.com 